$\begin{array}{ll}\text { Italique } & \text { Italique } \\ \text { Poésie italienne de la Renaissance }\end{array}$

XV | 2012

Varia

\title{
Un idiografo recuperato del Podere e della Balia di Luigi Tansillo
}

\section{Daniela lovino}

\section{OpenEdition}

Journals

\section{Edizione digitale}

URL: http://journals.openedition.org/italique/359

DOI: 10.4000/italique.359

ISSN: 1663-4438

\section{Editore}

Librairie Droz

\section{Edizione cartacea}

Data di pubblicazione: 1 dicembre 2012

Paginazione: 217-231

ISBN: 978-2-600-01639-1

ISSN: 1423-3983

\section{Notizia bibliografica digitale}

Daniela Iovino, « Un idiografo recuperato del Podere e della Balia di Luigi Tansillo », Italique [Online], XV | 2012, online dal 01 décembre 2015, consultato il 19 avril 2019. URL : http:// journals.openedition.org/italique/359 ; DOI : 10.4000/italique.359 
D A N IELA IOVINO

U N I D I G R A F O R E C U P ER A T O

DEL PODERE E DELLA BALIA

D I L U I I T A S I L L O 



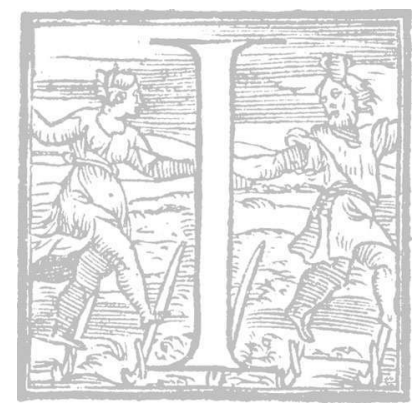

l manoscritto N. VII. 4 della Biblioteca Nazionale Universitaria di Torino è testimone fondamentale di due poemetti didascalici in terza rima di Luigi Tansillo, Il podere $e$ La balia e come tale utilizzato da Flamini nel 1893 per la sua edizione dell'Egloga e i poemetti. ${ }^{1}$ Questi $i$ dati disponibili sulla tradizione dei due poemetti, legati alla fase del ripiegamento idillico-borghese del Tansillo, marito e padre premuroso dopo la stagione dell'eroismo lirico amoroso. ${ }^{2}$

I. Prima dell' edizione Flamini, i lettori avevano avuto a disposizione il testo dei due poemetti attraverso due editiones principes varie volte ristampate: nel 1767 La balia (Vercelli, presso il Panialis) e nel 1769 Il podere (Torino, Reale Stamperia), dovute, almeno per quanto riguarda il testo, alle cure dall'erudito vercellese Giovanni Antonio Ranza (I74I-I80I), ${ }^{3}$ all'epoca non ancora preso dagli entusiasmi della rivoluzione. ${ }^{4}$ Presentando La balia, Ranza offre ( descrizione molto sommaria del manoscritto utilizzato:

A me tocca per avventura la sorte di far comuni agli Amatori della miglior Poesia Italiana siffatti bellissimi Capitoli, di cui ora ne cominciano uscir due intitolati la Balia, ossia Esortazione alle Nobili Donne a volersi allattar elle stesse i lor figli. Erano questi, insieme a tre altri inscritti il Podere, posti col Vendemmiatore, e le Stanze in lode della Menta, in un codice del secolo XVI, che passò, non è gran tempo, in forestiere contrade; e dal quale io trassi la copia, su cui si è fatta quest'edizione esattissimamente, tranne l'ortografia, che si adattò all'uso corrente.

Ranza sembra convinto che il manoscritto capitato tra le sue mani sia, almeno per quanto riguarda il contenuto, da identificarsi con gli «alcuni Capitoli dell'istesso Signor Tansillo bellissimi» ${ }^{6}$ dati come prossimi alle stampe da Barezzo Barezzi nell'avviso a' lettori che accompagna l'edizione veneziana (I606) delle Lagrime di san Pietro: progetto rimasto poi senza esito. ${ }^{7}$ Di questo testimone, tuttavia, allo stato non sembrano rimanere tracce.

L'attendibilità dell'antigrafo delle edizioni curate da Ranza si fonda sulla lettera di invio (datata Napoli, I gennaio I566), che accompa- 
gnava l'offerta dei due 'poemetti', a mons. Antonio Scarampo, che fu vescovo di Nola dal I549 al I569. Scarampo sarebbe stato nominato da Tansillo suo esecutore testamentario e tutore del figlio Mario Antonio: ${ }^{9}$ nel I 566 Tansillo fa omaggio all'amico vescovo delle due ultime operette composte (Le lagrime di san Pietro erano ancora in cantiere $e$ sarebbero rimaste alla morte dell'autore ancora bisognose di una revisione finale), ${ }^{ }{ }^{\circ}$ senza tuttavia eliminare la lettera di dedica del Podere a Giovan Battista Venere, che risaliva all'agosto del I $560,{ }^{\text {I }}$ sicché l'edizione Ranza della Balia propone in premessa ai versi la lettera a mons. Scarampo, mentre l'edizione del Podere è preceduta dalla dedica al Venere, maggiordomo di casa Piccolomini-d'Avalos, duchi di Amalfi. Il tenore della lettera testimonia il livello di confidente amicizia che si era instaurato negli anni tra il poeta e il prelato, mandato a Nola in giovane eta ${ }^{\mathrm{I} 2}$ come coadiutore dell'anziano vescovo Giovan Francesco Bruno, ${ }^{3}$ e dovrebbe costituire una garanzia sulla affidabilità del testo edito da Ranza:

Due cose io dono a V. S., delle quali ella ha poco, anzi niente bisogno; il Podere, e la Balia. Non ha bisogno dell'uno, perché mercé di Dio, e del suo merito, ha tante delle possessioni, che omai quasi le danno più fastidio, che comodo. Né men dell'altra ha mestieri, perché essendo V. S. in queste nostre parti il vero esemplare del buon Prelato in ogni virtù, e sopra tutte nella Castità, non ha figli, come altri forse, da porre a petto di Balia. [...] Li do a V. S. per ubbidirle, perché più volte me l'ha comandato; ed a questi giorni a Iano consegrati, ne' quali tra' padroni, e servitori, ed amici si dan vicendevolmente de' presenti, e come dicono in Roma, le mancie, qui le offerte, in Puglia le strene (benché sia del Latino) ed in Spagna l'aghinaldo, V. S. m'ha mandato de' frutti della sua ricchissima Diocesi, uve, e mela, che pajono or ora spiccate de su le viti, e da su gli arbori; e capponi, e copete: ed io le do quelli del mio povero giardino, o più tosto orticello, rime basse, e versi giocosi. Qualche dì forse le presenterò di que', che converranno al mio obbligo, ed al suo merito.

Questa lettera è uno dei documenti più tardi sottoscritti da Tansillo, che, meno di due anni dopo, nel fare testamento, memore della promessa fatta volle affidare a mons. Scarampo, insieme alla cura dell'unico figlio maschio, anche la decisione finale circa l'utilizzazione del suo lascito poetico: 
Item quod scripturae omnes tam de sua manu propria quam aliena, non tangantur ab alio et sit quivis, nisi ab Ill.mo et Re.mo Episcopo Nolano iuncto cum Ecc.mo Baldassarre de Torres si fuerit hic; si non fuerit, totum arbitrium sit ditti Re.mi Episcopi. ${ }^{\mathrm{I}}$

Ci troviamo di fronte a un insieme di elementi che, pur in assenza del manoscritto, dovrebbero delineare un quadro di affidabilità, perché sembra difficile immaginare che Tansillo potesse fare omaggio all'amico vescovo di un testo poco sorvegliato. ${ }^{\text {Is }}$

2. Francesco Flamini poté giovarsi per la sua edizione di un testimone altrettanto autorevole, il ms. N. VII. 4 della Regia Biblioteca Nazionale di Torino, che compensava la perdita del ms., che per comodità possiamo denominare "Scarampo", e che rispetto alle edizioni Ranza risarciva due lacune abbastanza vistose delle edizioni settecentesche. La descrizione offerta dallillustre studioso è alquanto parsimoniosa $e$ conviene rileggerla:

Cod. N.VII.4 della R. Biblioteca Nazionale di Torino, cartaceo, del sec. XVI, di carte 64 numerate, oltre due bianche e al frontespizio. In questo leggiamo: Capriccio di Luigi Tansillo | intitolato il Podere | partito in tre capitoli | Al gentiliss. et accortiss. | signor Giovan Battista | Venere. Tien dietro la dedicatoria al Venere [...] (p. CLIV)

Due pagine più avanti, nella sezione dedicata alla tradizione della Balia, si aggiunge:

Cod. N.VII.4 della R. Biblioteca Nazionale di Torino, descritto sopra. A carte 43r. Capriccio di Luigi Tansillo | detto La Balia | nel quale si essortan le donne nobili che elle | stesse si debban allattar | lor figliuoli | partito in duo cap.

Dal che si deduce che ci troviamo di fronte a una tradizione a due rami, facenti capo, uno all'antigrafo utilizzato dal Ranza, finito per ora su un binario morto, l'altro al codice torinese. Che si tratti di due testimoni tra loro indipendenti si può dedurre dal fatto che siano stati offerti in tempi diversi ai loro destinatari. Tuttavia i rapporti tra $i$ due testimoni, il torinese e l'antigrafo perduto, descritto però dalle edizioni del $1767 \mathrm{e}$ I769, saranno oggetto di approfondimento in vista della nuova edizione. Flamini (p. CLV) segnalò il comportamento poco attento dell'editore del Podere (non più Ranฉa, ma un «libraio torinese» non identificabile), 
che «non si era accorto d'una lacuna ragguardevole, di quattro terzine, ch'è a p. I2 della stampa da lui curata».

Il passo incriminato investe $i \mathrm{vv}$. 192-200 del primo capitolo (in corsivo $i$ versi mancanti alla princeps):

Non è sì scarsa o povera Natura, che ambedue grazie un loco aver non possa; ch'ella ba di noi, più che noi stessi, cura.

Fate pur nel cercarle vostra possa; oh quanti e quanti se ne veggono oggi, che comprando il poder compran la fossa!

Però desio che sian colline o poggi il sito ove le mura fondo et ergo, ma che per strada agevole si poggi:

benché spesso il mal aere d'un albergo si toglie col mutar d'usci e fenestre e far, ch'ove egli ha il petto, volga il tergo

(FLAMINI, pp. 204-05).

Anche per quanto riguarda la princeps della Balia Flamini lamentava ( $p$. CLVII) alcune lacune, che si erano poi trasmesse a tutte le edizioni successive. In questo caso, però, sembrava attribuirne la causa non a una distrazione dell'editore, ma alla responsabilità del copista cinquecentesco, che aveva «espunto due allusioni, delle quali l'una poteva sembrar lasciva, l'altra irriverente ( $v v .227-28$ del cap. I e 336 del cap. II)». Nel primo caso viene eliminato il riferimento al piacere legato all'atto della generazione umana:

Il generarlo [il figlio] vien senza fatica e non senza piacer, ben che 'l contrario da qualche una di voi talor si dica.

L'altra lacuna riguarda semplicemente il secondo emistichio dell'endecasillabo:

E 'n dirvi donne, intendo ogni persona del nobil sesso, et una non ne salvo, sia quantunque di cerchio o di corona.

In entrambi $i$ casi (pp. I3 e 54) Ranza annota: "Manca nel Manoscritto» e può essere una prova ulteriore che la lacuna più vistosa 
denunciata dalla princeps del Podere si debba ricondurre al responsabile della stampa e non al copista. ${ }^{16}$

Si può notare che nella descrizione del testimone torinese Flamini si limiti a collocarne la datazione al XVI secolo, senza spendere alcun riferimento alla grafia, o alla distribuzione dei versi nelle singole carte. Dal che si deve dedurre che a suo giudizio il ms. fosse stato copiato da un'unica mano.

Qualche anno dopo l'edizione, e precisamente nel gennaio I904, un incendio distrusse un numero notevole di manoscritti della biblioteca torinese e tra le vittime illustri si può ricordare anche il 'canzoniere' di Matteo Bandello. ${ }^{17}$ Stessa sorte sembrava essere toccata al ms. del Podere e della Balia tansilliani, la cui collocazione (N. VII. 4) era abbastanza prossima al ms. delle rime di Bandello (N. VII. $7 I) .{ }^{18}$

Prima di arrivare all'oggetto specifico di questa nota, ricordo che il ms. di Tansillo è riportato nell' antico catalogo a stampa della Regia Biblioteca di Torino: ${ }^{19}$

CODEX CLXIV. k. I. 95 N. VII. 4

Chartaceus, constans foliis 64. saeculi XVI. Capriccio di LUIGI TANSILLO intitolato il Podere partito in tre capitoli.

La segnatura K.I.9s è quella del precedente Inventario del Bencini, ${ }^{20}$ mentre N.VII.4 è la collocazione dell'epoca del Pasini e attuale.

Dovendo operare una ricognizione della tradizione manoscritta e a stampa dei cosiddetti "poemetti" di Tansillo, ${ }^{2}$ bo chiesto notizie alla direzione della Biblioteca Nazionale Universitaria di Torino sulla sorte toccata al ms. N. VII. 4. Ho avuto cosi modo di appurare che a seguito dei necessari restauri il ms. era tornato consultabile a partire dal 1969, sebbene le tracce dellincendio siano ancora ben visibili. ${ }^{22}$ Quasi tutte le pagine appaiono infatti irreversibilmente bruciate sul margine destro $e$ in alcune di esse sono diventate illeggibili le sillabe finali dei versi; per effetto del calore, inoltre, la carta e l'inchiostro si sono scuriti. Tuttavia con l'ausilio del testo di Flamini il testo è recuperabile senza difficoltà. Le carte più danneggiate sono, spiegabilmente, le iniziali e le finali. Riproduco il frontespizio, chiudendo tra parentesi quadre le sillabe attualmente scomparse per effetto del fuoco: 


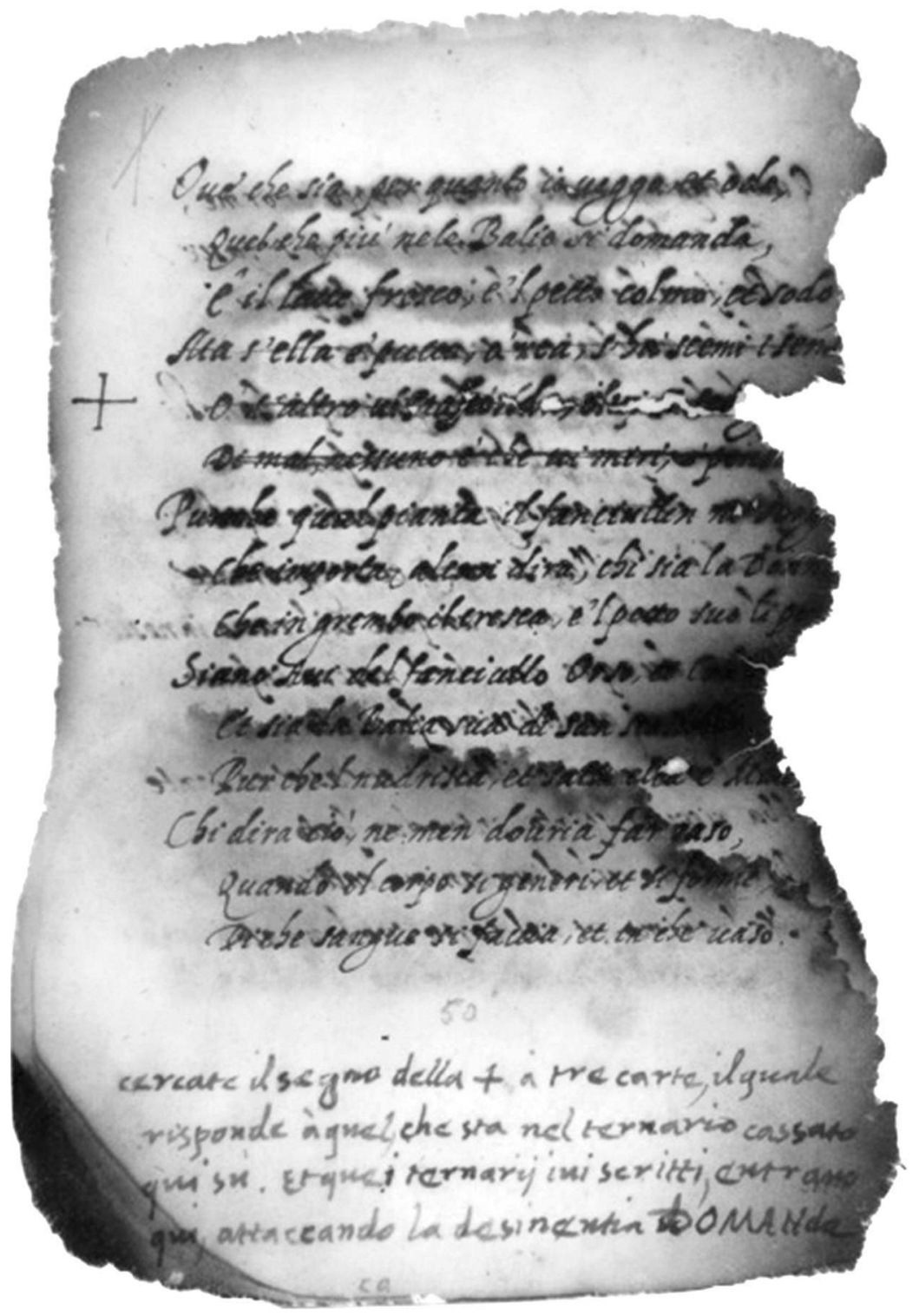

Photo I.

Capriccio | Di Lvigi Ta[nsillo] | Intitolato IL PODE[RE] | PARTito in tre CA[Pitoli] | [SPAZio Bianco] | Al gentiliss: ET ACCO[RTiss:] | Signor GiOVAN BATT[ISTA] | VENERE. | | 
A una prima verifica complessiva del testimone si è inoltre fatto strada il dubbio che Flamini non ne abbia preso diretta visione, poiché non si è accorto (o almeno non dà notizia) che le mani di scrittura sono due: la prima è quella di un copista di professione che in corsiva calligrafica ricopia il testo, evidenziando il capoverso di ogni terzina attraverso la collocazione fuori margine; la seconda, più approssimativa e sgraziata, interviene due volte sul testo della Balia, prima a c. sor e poi a c. 52v. Nel primo caso (foto I) ci troviamo verso la fine del primo capitolo della Balia e i vv. 238-40 appaiono barrati da una linea, con un segno di croce sul margine che rimanda ad un'annotazione a piè pagina: ${ }^{23}$

Ove che sia, per quanto io veggo et odo, quel che più ne le Balie si domanda è il latte fresco e 'l petto colmo et sodo,

Ma s'ella è putta o rea, s'ha scemi $i$ sensi, e s'altro vil nasconde, $[\ldots \ldots \ldots . . . . . . . .]^{24}$ di mal, nessuno ì che vi miri o pensi<.>

Purché qual pianta il fanciullin ne sorga, che importa, alcun dirà, chi sia la donna che in grembo il cresca, e 'l petto suo li porga?

La nota è la seguente:

cercate il segno della $\nmid$ a tre carte, il quale | risponde a quel, che sta nel ternario cassato | qui sù. Et quei ternarij ivi scritti, entrano | qui, attaccando la desinentia DOMANda. |

In effetti alla c. 52v, originariamente bianca, - ac. 52rdopo ilv. 319 ( «Riposiamoci un poco, et torniam poscia.») si legge: «IL FINE DEL P. CAP:》, mentre l'inizio delsecondo capitolo de La Balia è ac. $53^{r}$-troviamo I versi scritti dalla stessa mano che ha vergato la nota di c. sor, da leggere (foto 2) come integrazione a quelli cassati in precedenza:

Et si prende egualmente, et d'ogni banda, ove si trovi, et spesso a prender viensi per un vil servitor, che a ciò si manda.

Et s'ella è putta ${ }^{25}$ o rea, s'ha scemi sensi, o s'altro ella ha di mal, quando si piglia, nessuno è che vi miri o che vi pensi.

S'è bianca, o bruna, o pallida, o vermiglia, e 'n complession, che ben si mostra al viso, è contraria a la madre, o le somiglia. 


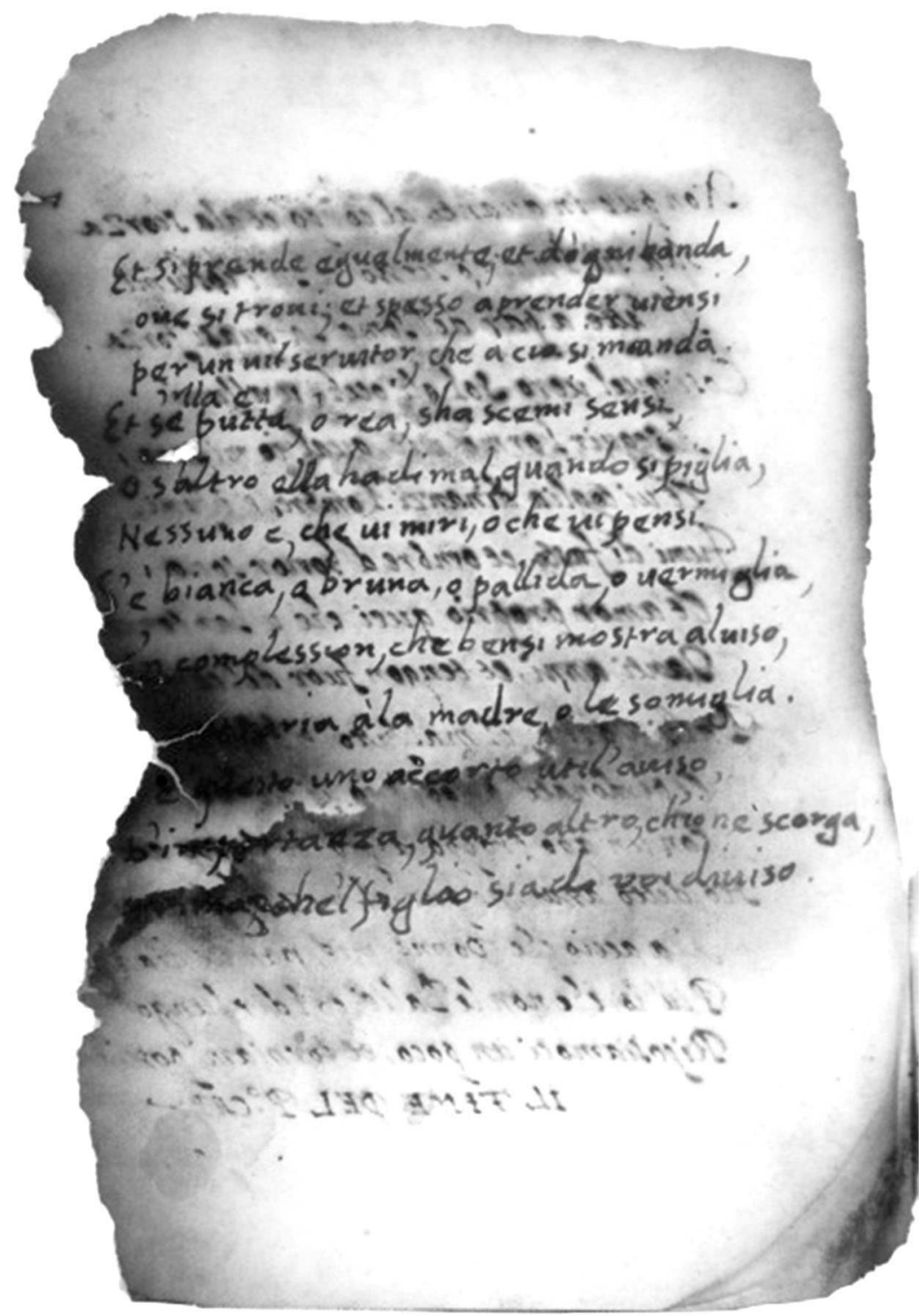

Photo 2.

Et è questo uno accorto util'aviso, d'importanza, quanto altro, ch'io ne scorga, prima che 'l figlio sia da voi diviso.

Giovandomi del recente contributo di Toscano sugli autografi di Tansillo $^{26}$ e ponendo a confronto la grafia della seconda mano del ms. $N$. 
VII. 4 con i campioni di scrittura autografa riprodotti fotograficamente si puo affermare senza ombra di dubbio che tanto l'avvertenza di c. sor, che $i$ I2 versi aggiunti a $c$. $52 \mathrm{~V}$ sono il frutto di un intervento correttorio dell'autore.

Flamini non cita questo repentino cambio di grafie, né tantomeno l'aggiunta dei dodici versi, e ciò induce a ipotizzare che, come era abbastanza consueto nella prassi filologica dell'Ottocento, egli si sia fatto trascrivere il manoscritto da qualche amico o collega studioso che aveva accesso alla Reale Biblioteca di Torino: l'anonimo trascrittore, evidentemente, si è limitato a seguire le indicazioni dell'allora non identificato postillatore senza segnalare altro.

Sembra difficile immaginare che a un occhio esperto come quello di Flamini potesse sfuggire un cosi vistoso cambio di mano e sicuramente avrebbe potuto di li sciogliere anche il dubbio circa la sottoscrizione della lettera di dedica che accompagna il manoscritto della Clorida (allora XII. o della Biblioteca Oratoriana dei Gerolamini di Napoli), caso in cui si limita a dire ( $\not . C X L)$ che «la data di questa [dedica] Napoli a' $x x$ di Febr. del xl vii, è, a partir dal $x x$, di mano diversa $e$ posteriore, d'inchiostro diverso $e$ in carattere corsivo; probabilmente, autografa».

Ancora oggi rimangono da chiarire alcuni elementi materiali circa la "ricostruzione" del manoscritto di Torino, poiché a seguito del restauro parte delle carte del primo e del secondo capitolo del Podere non appaiono collocate correttamente. ${ }^{27}$

Il recupero tuttavia appare di notevole importanza perché ci consente di aggiungere alla tradizione complessiva delle opere di Tansillo un manoscritto idiografo da affiancare ai pochi già noti, come il ms. 9-2I60 (olim I2.II.I, n. Is) della biblioteca della Real Academia de la Historia di Madrid, ${ }^{28}$ e il già citato codice della Clorida della biblioteca Oratoriana di Napoli. Ciò conferma oltre tutto la validità della scelta editoriale di Flamini. In aggiunta si può solo stabilire con certezza che il codicetto torinese deve essere considerato la copia di invio realizzata, come impongono di credere gli interventi correttori autografi, sotto il diretto controllo dell'autore. 
Il presente contributo è estratto dalla tesi di laurea magistrale in Letteratura italiana (L'egloga e i poemetti di Luigi Tansillo. Studio preliminare sulla tradizione manoscritta e a stampa: testi e apparati) da me discussa nell'ottobre $20 \mathrm{I}$ o nell'Università di Napoli "Federico II", relatore Tobia R. Toscano, che ringrazio per avermi inserita nel gruppo di lavoro per la realizzazione dell'edizione critica delle opere di Luigi Tansillo.

I. Napoli [ma Trani, Vecchi], I 893: d'ora in avanti: Flamini, seguito dal numero di pagina.

2. Secondo T. R. Toscano, Per una collocazione storica dell'esperienza lirica tansilliana, in Luigi Tansillo, Rime, introduzione e testo a cura di T. R. Toscano, commento di E. Milburn e R. Pestarino, Roma, Bulzoni Editore («Europa delle Corti» I 54), $20 \mathrm{I}$, vol. I, pp. I 8 I-87, il matrimonio (I 550 ) segna una cesura nell'attività poetica del venosino, che negli anni a seguire compose per lo più liriche di occasione o di tematica familiare e religiosa. Esaurita la fase più creativa del versante lirico, Tansillo tornò in qualche caso sulle sue rime solo per migliorarne la lezione. Flamini, p. XCVIII, ritiene la Balia «composta senza dubbio dopo il i 552 ( $\mathrm{ma}$ in quale anno precisamente non sappiamo)», mentre per il Podere il terminus ante quem è il is agosto i 560 , data che si legge in calce alla lettera di dedica a Giovan Battista Venere. Sembra però lecito ritenere che Tansillo vi stesse lavorando da tempo e che infine cedesse alle insistenze del dedicatario per non sembrare scortese: «Non vi meravigliate, ch'io m'abbia tanto indugiato a darlovi, sì spesso da voi essendomi richiesto; perciò che, quanto più ho veduto voi arder di desiderio d'averlo, tanto più io mi son venuto raffreddando di darlo; timido, che cosa fatta quasi da scherzo non mi recasse biasmo da dovero, dove fosse da voi avuto in troppa stima».

3. Laureatosi a Torino nel 1764 , Ranza era professore di umanità nelle regie scuole di Vercelli quando stampò la princeps della Balia, modernizzando la grafia secondo gli usi del XVIII sec. e «illustrandolo con molto amore, non senza una qualche larghezza di note dichiarative e di raffronti» (FLAminI, p. CLVII). Diverso il caso del Podere per il quale Ranza è da ritenersi responsabile solo del testo, ma non del commento, perché, secondo informa Bonaventura Porro, compositore della Reale Stamperia, nell'avviso che precede la princeps torinese, «aveva ceduto i capitoli del podere a un libraio di Torino, acciocché desse opera che venissero pubblicati», dopo aver abbandonato il progetto «di dar fuori le sue note».

4. Ranza è molto noto agli studiosi del giacobinismo italiano. Ebbe una vita molto movimentata: «Chi conobbe Giovanni Antonio Ranza nell'abito talare di professore delle Regie Scuole di Vercelli, o nell'abito laico di tipografo-editore, apprezzatissimo dall'élite culturale piemontese, mai avrebbe potuto supporre che un giorno avrebbe oscurato la non modesta rinomanza che s'era creata con l'ausilio delle divine muse, divenendo un rivoluzionario famoso, un turbolento vigilato dalle polizie di mezza Europa»: così R. Ordano, Figure, Vercelli, S.E.T.E., I977, pp. 7-IO, che ricorda anche la sua attività di fondatore e gestore della "Tipografia patria" da cui uscirono, tra il 1777 e il I 790 , oltre 200 libri e opuscoli, dall'«orientamento ideologico tipico dell'illuminismo, opposto a quello conservatore e clericale della tipografia Panialis, che allora stampava a Vercelli». Quindi il "giovane" Ranza, ancora prete e professore, mostrò un notevole interesse per la 
poesia idillico-didascalica di Tansillo, stampando presso il "conservatore" Panialis la Balia cedendo successivamente ad altri l'incombenza di pubblicare il Podere.

5. Ranza aggiunge in nota a questo passo che i capitoli del Podere «sono già illustrati con Note, ed usciranno fra pochi mesi, con isperanza di non minore aggradimento della Balia, per la molta varietà, con la quale seppe l'Autore illeggiadrire la secchezza e sterilità dell'argomento».

6. Perciò la sottolineatura bellissimi Capitoli dovuta a Ranza, che si riallaccia, citandolo, all'avviso di Barezzi.

7. Cfr. Luigi Tansillo, Capitoli giocosi e satirici, a cura di C. Boccia e T. R. Toscano, Roma, Bulzoni Editore («Europa delle Corti» I53), 2010, p. 71, nota, dove si ipotizza che il ms. dei capitoli tansilliani in possesso del tipografo Barezzi potrebbe avere semmai qualche parentela con il testimone M (Mss. Italiani cl. IX, cod. I74, coll. 6283, olim CIV.8 della Biblioteca Nazionale Marciana di Venezia: per la descrizione, cfr. pp. 54-56).

8. Stampata alle pp. V-VII della princeps e riproposta anche da Flamini, p. xcIC.

9. Cfr. Toscano, Premessa a Tansillo, Rime, ed. cit., p. 9.

ıo. La complicata vicenda editoriale del poema religioso tansilliano, passato prima per la revisione di Giovan Battista Attendolo ( 585 ) e successivamente di Tommaso Costo (i 606), è ricostruita da T. R. Toscano, Note sulla composizione e la pubblicazione de «Le lagrime di San Pietro» di Luigi Tansillo (con inediti), in Rinascimento meridionale e altri studi (raccolta di studi pubblicata in onore di Mario Santoro), Napoli, S. E. N., I987, pp. 437-6r.

I I. Si legge infatti alle pp. [7]-[8] della princeps del Podere.

I 2. Al momento della sua nomina a vescovo coadiutore di Nola ( 546 ) aveva 30 anni e viene ricordato dalla storiografia nolana come «un de' più zelanti e memorandi Vescovi di Nola»: G. Remondini, Della nolana ecclesiastica storia, Napoli, Giovanni Di Simone, t. III, I757, p. 216, citato da T. R. Toscano, Giovan Battista Attendolo editore di Luigi Tansillo: dalla princeps delle Lagrime di san Pietro (I585) al progetto non realizzato di una stampa delle Rime, in ID., L'enigma di Galeazzo di Tarsia. Altri studi sulla letteratura a Napoli nel Cinquecento, Napoli, Loffredo, 2004, pp. 2 I o-I I, che ricostruisce una succinta biografia di questo rigoroso interprete delle strategie tridentine (fondazione del seminario diocesano, appoggio ai gesuiti nella fondazione del collegio di Nola), che negli anni giovanili tuttavia non aveva mancato di leggere un libro "eretico" come il Beneficio di Cristo «del qual se agradaba mucho».

I 3. Resse la diocesi di Nola dal i 505 al i 549 , negli anni in cui si venne completando il cantiere della cattedrale e per il quale si avvalse dell'opera di scultori come Giovanni da Nola e Girolamo Santacroce, commissionando il polittico per l'altare maggiore ad Andrea da Salerno: cfr. la nota di Toscano al son. 3 delle Rime dubbie di Tansillo, ed. cit., p. 971, in cui si affaccia l'ipotesi che il sonetto tansilliano Che cosa è Dio? Rispondi: È un sempre bene, molto simile al son. Che cosa è 
Dio? Egli è un sommo bene, tràdito dal ms. 2620 della Biblioteca Universitaria di Bologna con attribuzione a mons. Bruno, sia la traccia di una condivisa variazione sul son. di Soneto in dialogo de Dio («Che cossa è Dio egli è un summu bene») di Bartomeu Gentil (stampato nel Cancionero general, Toledo, Ramon de Petras, I $527, \mathrm{c} . \mathrm{XVI} r$ ), risalente «al periodo in cui il giovane Tansillo tornava spesso a Nola (basti la testimonianza autobiografica affidata ai Capitoli I-III)».

I 4. Il testamento fu sigillato il 28 novembre $\mathrm{s} 568$, Tansillo morì due giorni dopo: il passo è riportato da Toscano, in Tansillo, Rime, ed. cit., p. 9.

I 5 . Bisogna però dire che il «codice del secolo XVI» passato per le mani di Ranza conteneva anche il testo del Vendemmiatore (il poemetto uscì con tale titolo e aumentato nel numero delle stanze, passate da 79 a I7I, nel i 549 a Venezia presso Baldassarre Costantini) e delle Stanze in lode della menta, che non sono di Tansillo (cfr. Flamini, pp. cxxxIv-xxxv): lettura non proprio consigliabile per un prelato come mons. Scarampo, rammentando che proprio il poemetto giovanile aveva fatto finire all'Indice dei libri proibiti del i 559 tutta la produzione di Tansillo, poi "assolta" dall'Indice del i 564 . Può darsi che il "codice" nella disponibilità di Ranza sia stato assemblato in una fase successiva a quella dell'offerta a Scarampo dei due poemetti in terza rima e che il nuovo possessore abbia fatto rilegare in unico codice opere di provenienza diversa. Né si può escludere che il testo del Podere e della Balia sia una copia ricavata dal ms. inviato al Vescovo di Nola nel i 566 .

I6. Delle due lacune, sembra che almeno la prima possa essere frutto di un'autocensura in un testo destinato a un vescovo.

i7. Cfr. Matteo Bandello, Rime. Edizione e commenti a cura di M. Danzi, Modena, Edizioni Panini, 1989, p. 326.

I 8. Tra le altre 'vittime' dell'incendio va ricordato anche il cod. CCLII. N. V. 4I, che leggeva il son. Sú, collige sarcinulas, Pacheco (n. 374, ed. cit. delle Rime di Tansillo, p. 886), fortunatamente trascritto da B. Peyron (Codices Italici manu exarati qui in bibliotheca Taurinensis Athenaei ante diem 26 ianuarii 1904 asservabantur recensuit, illustravit Bernardinus Peyron; praemittuntur C. Frati Italica praefatio et elenchus operum B. Peyroni typis impressorum, Taurini, apud Clausen, I904, pp. I $8 \mathrm{I}-2$ ), prima che il codice andasse distrutto.

19. F. Berta, A. Rivautella, G. Pasini, Codices manuscripti Bibliothecae regii Taurinensis Athenaei: per linguas digesti, [et] binas in partes distributi, in quarum prima Hebraei, [et] Graeci, in altera Latini, Italici, [et] Gallici, Ex Typographia Regia, I749, t. II, p. 452. 20. Francesco Domenico Bencini fu direttore della Biblioteca dal i 728 al I 732 . Il suo inventario manoscritto, Catalogo alfabetico dei manoscritti della Biblioteca della Regia Università di Torino, è datato I 729 c.a. Ringrazio la dott.ssa Franca Porticelli, responsabile Ufficio Manoscritti e Rari della Biblioteca Nazionale Universitaria di Torino, per le notizie fornitemi con grande cortesia e competenza e da cui apprendo che nei precedenti Inventari, quello del Torrini («Inventario della Biblioteca Ducale del protomedico e bibliotecario Giulio Torrini») e quello, pure manoscritto, di Filiberto Maria Machet («Index alphabetique des livres qui se trouvent en la Bibliotheque Royale de Turin en cette annèe 17 I 3 sous le regne de 


\section{UN IDIOGRAFO RECUPERATO}

S. M. Victor Amè Roy de Sicile, et de Chipre Duc de Savoye, et des Montferrat, Prince de Piemont») non compare il manoscritto di Tansillo. Pertanto la sua registrazione nell'Inventario del Bencini ne certifica la presenza nei fondi della Biblioteca del Regio Ateneo al momento della sua istituzione (1723) o poco dopo, ma è difficile stabilire (manca la documentazione di quegli anni e l'incendio del 1904 ha tolto dai manoscritti superstiti le tracce materiali distruggendo legatura e fogli di guardia) per quali vie sia giunto e se fosse già presente nei fondi ducali.

2 I. Come è noto sotto tale denominazione vengono rubricati da Flamini tanto i poemetti in ottava rima (Vendemmiatore, Stanze a Bernardino Martirano, La Clorida. Stanze al Viceré Toledo) che quelli in terza rima (Il podere e La balia), che Tansillo denomina "capricci", con ciò ricollegandosi alla più abbondante e (prevalentemente) giovanile produzione di capitoli giocosi e satirici.

22. Ho condotto questa prima ricognizione sulla scorta di una copia digitale su CD ROM.

23. Le trascrizioni che seguono riproducono senza interventi la grafia del manoscritto. Il corsivo segnala lezioni che a distanza di due carte appaiono modificate.

24. L'acidità dell'inchiostro ha bucherellato la carta, rendendo illeggibile la parte finale del verso.

25. Inizialmente Et se putta, con aggiunta in interlinea del segno di apostrofo su se seguìto da lla è.

26. T. R. Toscano, Luigi Tansillo, in Autografi dei letterati italiani. Il Cinquecento, tomo I, a cura di M. Motolese, P. Procaccioli, E. Russo, Roma, Salerno editrice, 2009, pp. 319-25.

27. Attualmente le carte presentano in basso al centro una numerazione recente a matita, mentre nella parte alta delle carte non sembrano rimanere tracce della numerazione originaria. Lo scorrimento regolare del testo corrisponde all'attuale numerazione delle carte: cc. I-I4, 22 (cap. I); 21, I7-20, I6, I 5, 23-28 (cap. II). Tanto il cap. III del Podere che i due capp. della Balia non presentano irregolarità nella cartulazione. Si osservano inoltre parole di richiamo in fondo alle cc. $24 \mathrm{v}$, $36 \mathrm{v}$ e $53 \mathrm{v}$. Anche su questo aspetto sarà necessario un supplemento di indagine, perché l'intervallo non appare regolare: se si fosse trattato di fascicolazione a sesterni, il richiamo sarebbe dovuto comparire anche a c. I $2 \mathrm{~V}$ e a c. $48 \mathrm{v}$, mentre rimane poco spiegabile a c. 53v. Allo stato il ms. è composto di carte sciolte collocate in taschine trasparenti e non rimangono tracce della cucitura dei fogli.

28. Battezzato come autografo da E. Pèrcopo, che non ebbe mai modo di vederlo, si è rivelato poi a un controllo diretto un idiografo con correzioni autografe dell'autore: cfr. Toscano in Tansillo, Rime, ed. cit., pp. 3 I-33 e I I 9-27. 